\title{
Similarity metric in medical image registration
}

Jing Jin

School of Communication and Information Engineering University of Electronic Science and Technology of China Chengdu, China

elise_jing@163.com

\begin{abstract}
Registration algorithm via various similarity measures is presented in this paper. Image registration is a process of align images so that they can get the same structure in the same spatial locations. During the registration, similarity metric is very important to measure the result as a standard. Different similarity metric has its own advantages and application field. Mutual information, correlation ratio and least squares are mentioned in this paper. Problematic issues of these three methods and outlook for the future research are discussed too. The major goal of the paper is to provide a comprehensive comparison among the widely used similarity metric.
\end{abstract}

Keywords-registration; similarity metric; mutual information; correlation ratio; least squares

\section{INTRODUCTION}

Image registration is a process of finding the optimal transformation that aligns two or more images into spatial correspondence, so that we can get the same structure of the different images in the same spatial locations. Image registration is a very important step in image analysis tasks. For example, remote sensing, multi-modality information fusion, medical image processing, to name just a few.

During the past two decades, a large number of image registration methods have been developed [1]. Because of the diversity of images and various types of degradations, it is impossible to design a universal method applicable to all registration tasks. Nevertheless, the majority of the registration methods consist of the following four steps: feature detection, feature matching, transform model estimation, image resampling and transformation. Each step has its problem to implement. According to the first step of image registration, methods can be classified into two main categories: area-based methods and feature-based methods. Feature-based methods often applied to images which contain a lot of details (towns, rivers, roads, forests, room facilities, etc.). Medical images are not so rich in details and thus area-based methods are usually employed here. No features are detected in area-based methods so the first step of image registration is omitted; these methods focus on feature matching step.

There are lots of similarity measures in matching step to measure the similarity between the images. In this paper, we compared three different similarity measures in MRI registration: mutual information [2], correlation ratio [3], and least squares [4]. In section 2, principles and basic function of these methods will be displayed. Different metric used in

\author{
Yunle Yang \\ School of Communication and Information Engineering \\ University of Electronic Science and Technology of China \\ Chengdu, China \\ uestcyyl@163.com
}

MRI registration based on free form deformation [6] will be described in section 3. Section 4 discuss about the advantages and disadvantages about the three methods. Advantages should be improved the accuracy of registration.

\section{SIMILARITY METRIC}

In image registration, $I_{r}(x, y)$ and $I_{s}(x, y)$ are the intensity of the reference image and sensed image. We want to find a transformation $\mathrm{T}$ that maps every voxel in sensed image to its correspondence voxel in reference image by maximize the similarity metric and minimized regularization or penalty term between two images.

$$
I_{r}(x, y)=I_{s}(T(x, y))
$$

During the last two decade, more and more similarity metric are used in image registration. They are the criterion of each transform. Mutual information, correlation ratio, least squares are often used in medical image registration.

\section{A. Mutual information methods}

The mutual information (MI) methods appeared recently and represent the leading technique in multimodal registration which is always a problem in registration field. Mutual information methods originating from the information theory, is a measure of statistical dependency between two data sets. MI between two random variables $A$ and $B$ is given by

$$
M I(A, B)=H(A)-H(A \mid B)=H(A)+H(B)-H(A, B)
$$

Where $H(A)=-\sum_{a} p_{A}(a) \log _{2} p_{A}(a)$ represents the entropy of $A$ and $p_{A}(a)$ is the probability distribution of $A \cdot H(A, B)=-\sum_{a, b} p_{A B}(a, b) \log _{2} p_{A B}(a, b)$ is the joint entropy of $A$ and $B$. In registration we can get $p_{A}(a)$ and $p_{B}(b)$ from the histogram of both sensed and reference image. $p_{A B}(a, b)$ can be calculated from the joint histogram of the two images. Nevertheless, in mathematical, 
we can get $p_{A}(a)=\sum_{b} p_{A B}(a, b)$, so we can compute all these probability distribution form joint histogram.

\section{B. Correlation ratio methods}

In statistics, the correlation ratio is a measure of the relationship between the statistical dispersion within individual categories and the dispersion across the whole population or sample. The measure is defined as the ratio of two standard deviations representing these types of variation.

In order to compute the correlation ratio between two images defined as random variables. A common technique consists of normalizing the image pair 2D-histogram. Then, the image may be seen as discrete random variables. The correlation ratio can be computed recursively by accumulating local computations. Let $\Omega$ denote the images overlapping region, and $N=\operatorname{Card}(\Omega)$ the total number of voxels it contains. We consider the sets of $X$, $\Omega_{i}=\{\omega \epsilon \Omega, X(\omega)=i\}$ and their cardinals $N_{i}=\operatorname{Card}\left(\Omega_{i}\right)$ The total and conditional moments (mean and variance) of $Y$ are:

$$
\begin{aligned}
& \sigma^{2}=\frac{1}{N} \sum_{\omega \in \Omega} Y(\omega)^{2}-m^{2}, m^{2}=\frac{1}{N} \sum_{\omega \in \Omega} Y(\omega) . \\
& \sigma_{i}^{2}=\frac{1}{N_{i}} \sum_{\omega \in \Omega_{i}} Y(\omega)^{2}-m_{i}^{2}, m_{i}^{2}=\frac{1}{N_{i}} \sum_{\omega \in \Omega_{i}} Y(\omega) .
\end{aligned}
$$

We can get a simple expression for the correlation ratio:

$$
1-\eta(Y \mid X)=\frac{1}{N \sigma^{2}} \sum_{i} N_{i} \sigma_{i}^{2}
$$

The algorithm derived from these equations does not require the computation of images 2D-histogram.

\section{C. least squares methods}

Least squares methods represent as follows:

$$
\sum_{i=0}^{N}\left\|p_{i}-q_{i}\right\|^{2}
$$

Where $p_{i}$ is a point in point set $\left\{p_{i}\right\}, i=1,2, \ldots, \mathrm{N} \cdot q_{i}$ is a point of point sets $\left\{q_{i}\right\}, i=1,2, \ldots, N$. In registration, we can get $\left\{p_{i}\right\}$ from reference image and $\left\{q_{i}\right\}$ from every transformed sensed image.

\section{MRI registration}

To comparing the results after registration by different similarity metric we mentioned above, we test brain MR images from different people so that the difference between the results of registration will be obviously (see fig. 1). In each experiment we use the same transform model: free form deformation (FFD) based on B-spline [7]. Finding the maximum of similarity measure is a multidimensional optimization problem, in this paper, we all use the gradient descent optimization method [2].

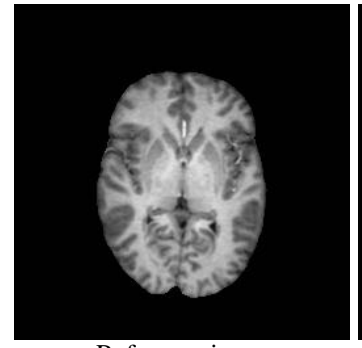

Reference image

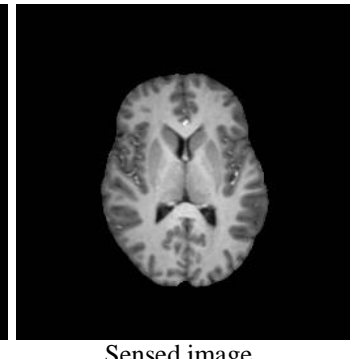

Sensed image
Figure 1. Examples of reference image and sensed image

\section{A. $\mathrm{T} 1$ to $\mathrm{T} 1$ registration}

In the test of $\mathrm{T} 1$ to $\mathrm{T} 1$ registration between different brain MRI, a big difference around the ventricle can be found in fig. 2. In three pictures, the outline of the brain are all like the reference image, but registration based on least squares and correlation ratio retain the most feature in the sensed image around the ventricle, while the method based on mutual information combine the characters between the reference image and the sensed image. Registration between the same modality, we can hardly find the superiority among the different similarity metric by our eyes, especially between correlation ratio and least squares. In fig. 3, differences are made in registration image and reference image.

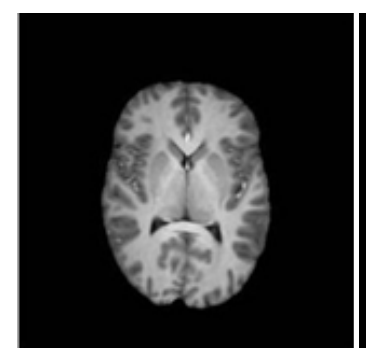

MI

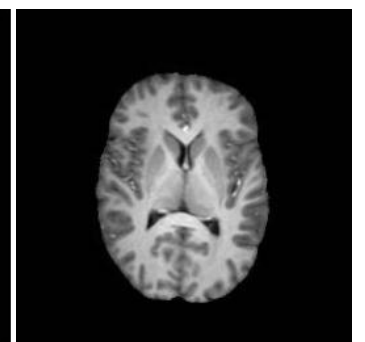

Correlation ratio

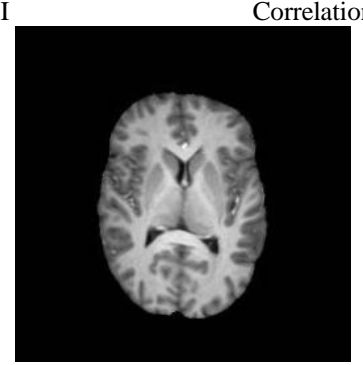

Least squares

Figure 2. T1 to T1 registration via MI, correlation ratio and least squares 

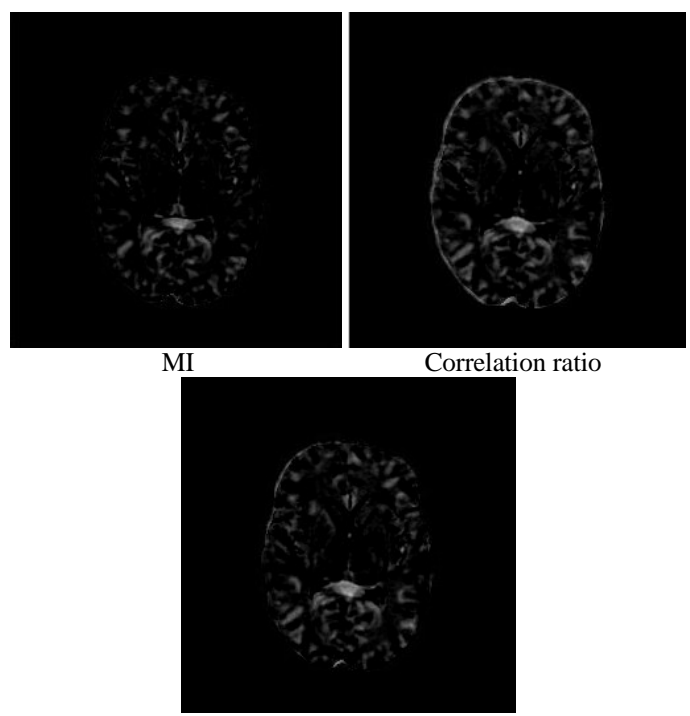

Least square

Figure 3. Difference between the $\mathrm{T} 1$ to $\mathrm{T} 1$ registration results and reference image

The results show us in fig. 3 that registration via MI is the most similar to reference image, especially the outline. The most lighted structure is the difference in the blow of the ventricle, which we can find it retains the character of the sensed image. By contrast, results after registration via correlation ratio and least squares have more difference in outline of the brain. In fig. 2 correlation ratio and least squares does not have obviously difference. But in fig. 3 least squares has outstanding performance in outline.

\section{B. T2 to $T 1$ registration}

The advantage of MI is obviously in fig. 4, which we take registration between T2 to T1 of different brain MRI. The pictures use correlation ratio and least squares similarity metric has serious distortion, especially the result use least squares. Fig. 5 displays the difference between registration results and T2 reference image. Lots of distortions have happened around the ventricle via correlation ratio and least squares similarity measure. The MI similarity measure gives a better registration.

Fig. 4 represents the results differ sharply from results in fig. 2, although the different type we use in registration, but the reference image and sensed image are all from the same one. The distortion in fig. 4 makes very bad results and it does not help us to define the location of the same structure. Fig. 5 represents clearly registration after correlation ratio and least squares give an incorrect location of ventricle between reference image and sensed image. Registration via MI takes a good performance.

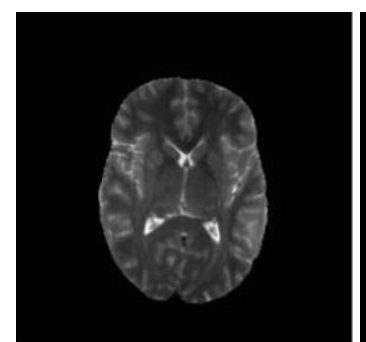

MI

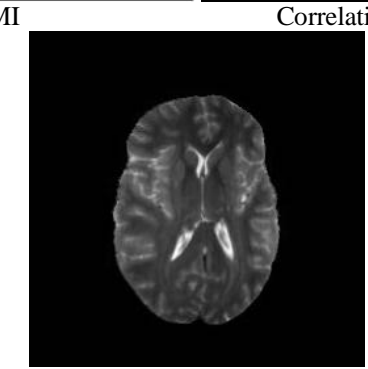

Least squares

Figure 4. T2 to T1 registration via MI, correlation ratio and least squares

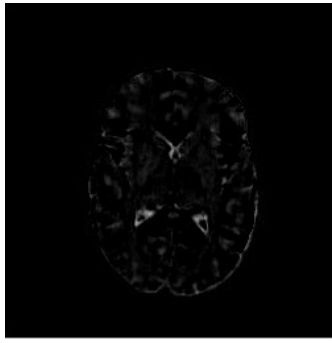

MI

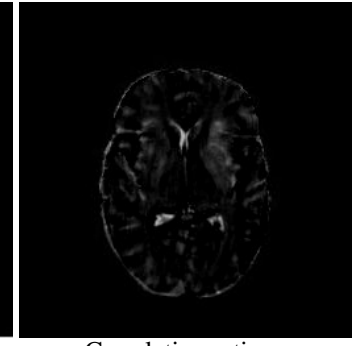

Correlation ratio

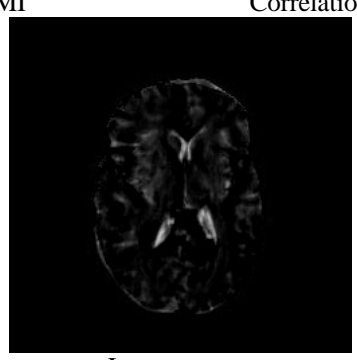

Least squares

Figure 5. Difference between the T2 to T1 registration results and reference image

\section{DISCUSSION}

As the result we tested above, MI methods have an advantage in multimodal registration. That is the reason that MI widely used in medical image registration. The three methods we discussed above are all area-based methods which have two principle limitations: reference image and sensed image must have somehow 'similar' intensity functions, either identical (and the correlation-like methods can be used) or at least statistically dependent (this typically occurs in multimodal registration).

In area-based methods, they often utilize all imaging data equally, which usually undermines the performance of the 
optimization process. Actually, different anatomical regions/voxels usually have different abilities to establish correspondences across images [5]. So recently, registration methods using simultaneously both area-based and featurebased approaches have started to appear. In the future, further to improve similarity metric, applying $\mathrm{MI}$ on extracted features is interested.

\section{REFERENCES}

[1] Zitova, B., Flusser, J., 2003. Image registration methods: a survey. Image and Vision Computing 21 (11), 977-1000.

[2] Pluim. J.P.W., Maintz, J.B.A., Viergever, M.A., 2003. Mutualinformation-based registration of medical images: a survey. IEEE Transanctions on Medical Imaging 22 (8), 987-1004.

[3] A. Roche, G. Malandain, X. Pennec, N. Ayache, The correlation ratio as a new similarity measure for multimodal image registration, Proceedings of the First International Conference on Medical Image
Computing and Computer-Assisted Intervention (MICCAI'98), Lecture Notes in Computer Science, Cambridge, USA, vol. 1496, 1998, pp. 1115-1124.

[4] PJ, Besl., ND, McKay, A method for registration of 3-D shapes. IEEE Transactions on pattern analysis and mechine intelligence, vol. 14, NO. 2, 1992.

[5] Yangming, Ou., A, Sotiras. DRAMMS: deformable registration via attribute matching and mutual-saliency weighting. Medical Image Analysis, 50-62, 21, 2009.

[6] S. Lee, G. Wolberg, and S. Y. Shin, "Scattered data interpolation with multilevel B-splines,” IEEE Trans. Visualization Comput. Graph., vol. 3, pp. 228-244, July 1997.

[7] Rueckert, D., Sonoda, L.I., Hayes, C., Hill, D.L., Leach, M.O., Hawkes, D.J., 1999. Nonrigid registration using free-form deformations: application to breast MR images. IEEE Transactions on Medical Imaging 18 (8), 712-721. 